\title{
Die komplexe Handverletzung
}

\author{
Alexander Zach, Axel Ekkernkamp, Andreas Eisenschenk
}

\section{Zusmmenfassung}

Unter einer komplexen Handverletzung versteht man die Zerstörung mehrerer funktioneller und sensibler Strukturen. Die Versorgung dieser Verletzungen stellt höchste Ansprüche an den Operateur, welcher versierte hand- und mikrochirurgische Kenntnisse besitzen muß. Die Rekonstruktion der Funktion der Hand steht an erster Stelle der Versorgung, der kosmetischen Aspektdarf aber nicht außer
Acht lassen. Neben dem Debridement ist die Versorgung aller Strukturen bei der Erstversorgung anzustreben. Ist dies nicht möglich, so sollte eine definitive Versorgung innerhalb von 72 Stunden erfolgen. Die krankengymnastische Nachbehandlung ist die 2. Säule in der Therapie der dieser Verletzung. Aufbauend auf die operative Versorgung entscheidet sie über den funktionellen Erfolg in der Behandlung einer komplexen Handverletzung.

\section{Einleitung}

Neben der großen Hirnmasse ist es auch die Hand, die den Menschen von allen anderen Lebewesen der Erde unterscheidet. Die Fähigkeit des aufrechten Ganges ermöglichte die Entwicklung eines Werkzeugs, welches nicht nur aufgrund seiner Kombination aus Kraft, Stabilität und Präzision einzigartig ist, sondern zudem auch noch Eigenschaften eines Sinnesorgans, wir nennen sie schlicht kognitive Diskrimination und taktile Gnosis, und die eines Sprachorgans besitzt. Mit ihr können nicht nur emotionale Signale empfangen, sondern auch gesendet werden. Es ist nicht nur möglich, mit der Hand Hör- und Sprachverlust in Form der Zeichensprache zu ersetzen. Blinden ermöglicht sie den Umgang mit der Umwelt, in der sogar das Lesen selbstverständlich ist. Die Hand ist neben dem Gesicht das einzige Körperteil, welches nahezu immer sichtbar ist. Hieraus resultiert eine enorm wichtige ästhetische Funktion. Kosmetische Schädigungen der Hand werden häufig emotional störender empfunden als funktionelle Verluste. Den Verlust eines oder mehrerer

OP-JOURNAL 2001; 17: 270-274

(c) Georg Thieme Verlag Stuttgart · New York
Finger an einer Hand z.B. kann man vollständig oder teilweise funktionell kompensieren, die Kosmetik einer solchen Verletzung ohne Epithesen z.B. nicht. Patienten mit geschädigten Händen haben sehr häufig psychische Probleme, was vom einfachen Verstecken der verletzten Hand bis hin zu Schwierigkeiten in der psychologischen und beruflichen Integration führen kann $[5,7]$.

Unter einer komplexen Handverletzung verstehen wir die Zerstörung mehrerer funktioneller und sensibler Strukturen (Knochen, Bänder, Sehnen, Nerven usw.) mit Verletzung der Weichteildeckung unterschiedlicher Ausprägung. Geschlossene Verletzungen sind von offenen zu unterscheiden. Geschlossene Handverletzungen sind äußerlich häufig unscheinbar, zeigen aber neben Frakturen und Luxationen oftmals eine erhebliche Traumatisierung der Weichteile mit Zerstörung von Nerven, Gefäßen, Sehnen, Bändern und nicht zuletzt der Handbinnenmuskulatur, welche sekundär nekrotisch werden kann. Gefäßschädigungen führen ebenfalls sehr häufig zu sekundären Nekrosen mit nachfolgendem Verlust wichtiger Strukturen. Offene Verletzungen weisen neben den oben genannten Verletzungen noch Defektverletzungen bis hin zu Amputationen und nicht zu- letzt hohe Verschmutzungsgrade mit hoher Keimkontamination auf. Die häufigsten Ursachen für solche Verletzungen sind Quetschungen der Hand und Kreissägenunfälle Abb. 1.u.2. Gerade Quetschungen oder Amputationen kommen dabei nicht selten im Rahmen eines Polytraumas vor. In solchen Fällen besteht nach wie vor der von Biemer geprägte Lehrsatz „Life before limb“ [2]. Allerdings ist das Vorliegen eines Polytraumas nicht zwingend eine Kontraindikation für eine Replantation. Nach Wilgis gibt es grundsätzlich nur noch zwei zwingende Kontraindikationen für eine Replantation. Zum einen die Ablehnung durch den Patienten und zum anderen eine vitale Bedrohung des Patienten durch eine Replantation [12].

\section{Die präoperative Phase}

Neben den allgemeinen OP-Vorbereitungen wie die Abklärung der Tetanusimmunisierung, welche bei nicht oder fraglichem Vorhandensein aufgefrischt wird, das Legen von venösen Zugängen und der Verabreichung eines Antibiotikums unterteilt sich die präoperative Phase in 4 Teile:

1. ausführliche Anamneseerhebung.

2. klinische Untersuchung

3. bildgebende Diagnostik

4. ausführliche Aufklärung

5. Festlegung des taktischen operativen Vorgehens

Neben der allgemeinen Anamnese und dem körperlichen Status des Patienten sollte eine präzise Unfallanamnese, soweit dieses möglich ist, erfolgen, da durch sie das Verletzungsausmaß sehr häufig schon grob abzuschätzen ist.

Gerade bei Verletzungen, welche äußerlich unscheinbar wirken (z.B. Hochdruckpistolen- oder Verletzungen mit Gas- oder Schreckschusspistolen), ist die Anamnese immens wichtig und kann präoperativ der einzige Hinweis auf das Verletzungsausmaß sein [10]. 


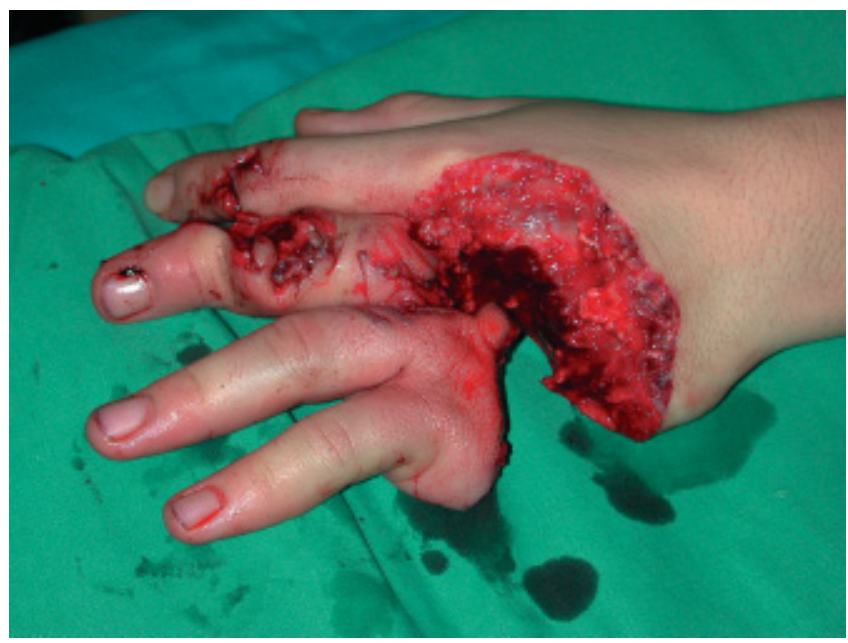

Abb. 1 Subtotale Mittelhandamputation

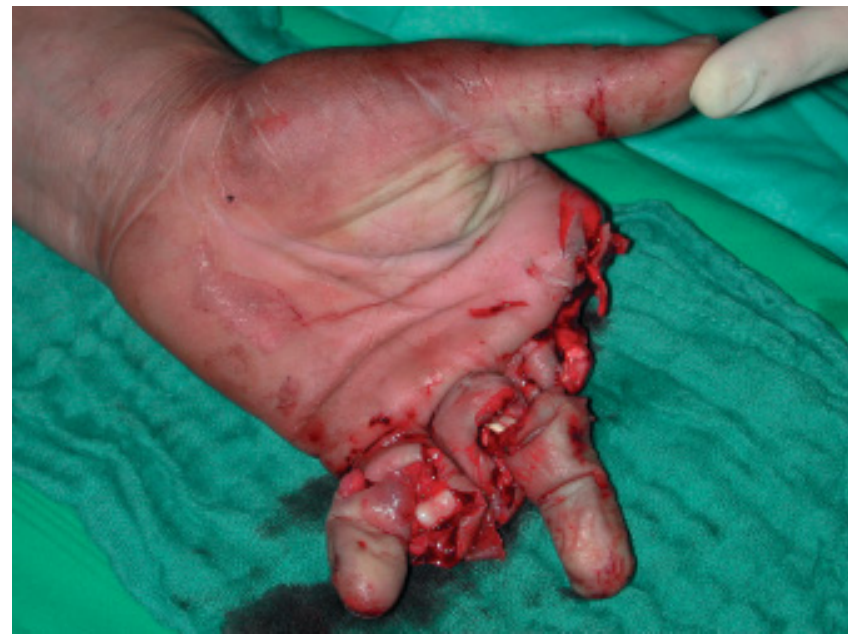

Abb. 2 Quetschverletzung
Weiterhin sollte ein besonderes Augenmerk auf die soziale und berufliche Anamnese gelegt werden. Die berufliche Anamnese schließt die Erfassung der tatsächlichen Tätigkeit des Patienten ein. So kann sich die Tätigkeit z.B. eines Büroangestellten auf ganztägiges Sitzen am Computer oder aber auch auf das ganztägige Herumtragen von Aktenordnern beziehen.

Im Anschluss daran erfolgen die Inspektion und die Funktionsprüfung der verletzten Hand. Sie umfasst die Durchblutungssituation, Motorik, Sensibilität, Stabilität des Skeletts und das Ausmaß der Weichteilschädigung. Während die Beurteilung der Durchblutung, der Knochenstabilität und des Weichteilmantels relativ patientenunabhängig sind, ist man bei der Prüfung der Motorik und der Sensibilität sehr auf die Mitarbeit des Patienten angewiesen. Die Voraussetzung ist hier ein ansprechbarer Patient. Aber selbst hier gestaltet es sich häufig sehr schwierig, das Verletzungsausmaß zu erfassen und sollte deshalb ausschließlich durch einen erfahrenen Handchirurgen erfolgen.

Zur Beurteilung der knöchernen Verletzungen wird eine Röntgendiagnostik der betroffenen Hand durchgeführt. Die Aufnahmen erfolgen üblicherweise in 2 Ebenen, wobei wir in unserer Klinik bei Verletzungen der gesamten Hand auch 3 Ebenen anfertigen lassen, da häufig die beiden angestrebten Ebenen unfallbedingt nicht korrekt erstellt werden können. Spezielle Aufnahmen wie z.B. eine Kahnbeinserie, Zielaufnahmen usw. sind eher die Seltenheit und meist nicht praktikabel. Dies gilt auch für den Einsatz der Computertomographie, welche sich nur in Einzelfällen im Notfall als sinnvoll erweist.

Die Versorgung einer komplexen Handverletzung berücksichtigt nicht nur die funktionellen, sondern auch die kosmetischen Gesichtspunkte der Hand. Dies muss für jeden Patienten individuell entschieden werden.

Die Rekonstruktion der Funktion der Hand steht bei der Erstversorgung an erster Stelle.

Der Patient selbst ist bei Einlieferung im Krankenhaus nur bedingt in der Lage, eine Entscheidung hinsichtlich seiner Versorgung zu treffen. Er kann aufgrund mangelnder Erfahrung seine Verletzung nicht einschätzen und befindet sich in einer extremen Stresssituation. Wichtig ist hier eine sehr ausführliche Aufklärung über die Möglichkeiten der Handrekonstruktion und über die Perspektiven des zu erwartenden funktionellen Ergebnisses (Beweglichkeit, Sensibilität Kosmetik). Diese müssen dem Patienten unter Berücksichtigung seines sozialen und beruflichen Umfeldes individuell erläutert werden. So sind die Anforderungen an die Hand z.B. einer Verkäuferin andere als die eines Maurers. Um eine solche umfassende Aufklärung durchzuführen und um anschließend mit dem Patienten Entscheidungen treffen zu können, muss der Chirurg in der Rettungsstelle über eine mehrjährige Erfahrung in der Behandlung solcher Verletzungen und der möglichen sekundären rekonstruktiven Maßnahmen besitzen. Es ist wichtig das Vertrauen des Patienten zu gewinnen, was nur durch eine qualitativ sehr gute Aufklärung vor der Operation zu erzielen ist. Die psychologische Führung des Patienten beginnt vor allem in der präoperativen Phase. Schwarzmalerei sollte hierbei tunlichst vermieden werden. Allerdings dürfen auch keine allzu großen Hoffnungen geweckt werden.

Die Gestaltung der präoperativen Phase richtet sich nach der Dringlichkeit der operativen Versorgung. Im Falle einer Perfusionsstörung oder replantationswürdigen Amputationsverletzung ist eine schnelle Versorgung zur Erhaltung der betroffenen Extremität vordergründig. Der zeitliche Aspekt ist häufig gerade in den Versorgungszentren, wo die Patienten nach erfolgter Erstversorgung in einem ortsansässigen Krankenhaus mit z.T. mehrstündiger Latenz eintreffen, ein großes Problem.

Am Ende der präoperativen Phase wird das operationstaktische Vorgehen festgelegt. Ausgehend vom Verletzungsausmaß muss hier nicht nur die Dringlichkeit der Versorgung berücksichtigt werden, sondern auch personelle und technische Faktoren.

\section{Die operative Versorgung}

Mit der operativen Versorgung wird der Grundstein der weiteren Behandlung gelegt. Die Qualität der operativen Erstversorgung ist maßgebend für das zu erwartende Ergebnis. Voraussetzung ist ein erfahrener Operateur mit versierten handund mikrochirurgischen Kenntnissen, ein entsprechend ausgerüsteter Operationssaal, ein präoperativ festgelegtes klares, taktisches operatives Konzept und nicht zuletzt ausreichend Zeit. Gerade bei Amputationsverletzungen kann der Einsatz 
von zwei Operationsteams, welche ergänzend arbeiten durch den zeitlichen Gewinn enorm zum Erfolg beitragen.

Anzustreben ist nach der Extremitätenerhaltung die definitive Versorgung aller verletzten Strukturen unter Berücksichtigung der Erfordernisse des Patienten.

Die Extremitätenerhaltung sollte immer unter dem Gesichtspunkt des zu erwartenden funktionellen Ergebnisses durchgeführt werden, denn eine funktionslose Hand ist im alltäglichen Leben auch bei gutem kosmetischen Ergebnis eher hinderlich und schon 1981 resümierte Chen: „Restoration without funktion is not success" $[1,4]$. Ist eine Versorgung von einzelnen Strukturen primär nicht möglich oder sinnvoll, erfolgt dies sekundär.

Die operative Versorgung beginnt nach Anlage einer Blutleere mit einer ausgiebigen Wundreinigung und sorgfältigem Debridement, in welchem gequetschtes und nekrotisches Gewebe konsequent entfernt wird.

Anschließend werden die zu rekonstruierenden Strukturen dargestellt und markiert. Hierzu ist es erforderlich das Operationsgebiet bis ins gesunde Gewebe zusätzlich weit zu eröffnen. Wurde die Ischämiezeit deutlich überschritten, muss die betroffene Muskulatur debridiert werden. Gewebe, dessen Vitalität nicht eindeutig beurteilbar ist, sollte zur Vermeidung eines unnötigen Verlustes frühsekundär beurteilt und dann debridiert werden. Gerade bei ausgeprägten Quetschverletzungen ist ein zwei- oder mehrzeitiges, kurzfristiges Vorgehen zu empfehlen. Das konsequente Debridement ist eine Grundlage zur Vermeidung eines postoperativen Infektes. Bei vielen Weichteilquetschungen und Weichteilverletzungen ist die Spaltung des Karpalkanals, der Loge de Gyone und der Faszien der intrinsischen Muskulatur obligat, um ein Kompartmentsyndrom zu vermeiden. Zuerst wird nun der Knochen stabilisiert.

Wichtig ist eine minimalinvasive, schnelle und stabile Osteosynthese vorzunehmen.

Sie wird häufig mit Kirschner-Drähten und/oder Cerclagen vorgenommen. Schrauben- oder Plattenosteosynthesen an der Hand sollten in seltenen Fällen bei derartigen Verletzungen nur ab der
Mittelhand nach proximal betrachtet zur Anwendung kommen. Das Ziel ist eine stabile, rotations- und achsengerechte Osteosynthese zur Erlangung einer frühfunktionellen Behandlung. Bei den Mittelhandknochen muss zusätzlich auf eine ausreichende Länge geachtet werden, was z.B. durch das Einbringen von transmetakarpalen Drähten ermöglicht werden kann. Die Voraussetzung bei diesem Vorgehen ist allerdings das Vorhandensein mindestens eines intakten Metakarpalknochens als Stützpfeiler. Die Alternative zu diesen Verfahren ist die Anlage eines Fixateur externe, welcher sich gerade bei ausgeprägten Weichteilquetschungen und knöchernen Defektverletzungen anbietet. Mit ihm ist eine sehr schnelle, minimalinvasive und defektüberbrückende, primäre Osteosynthese möglich, welche allerdings nicht immer eine exakte, rotations- und achsengerechte Skelettstabilisierung erzielt und eine frühfunktionelle Behandlung verhindert. Sehr häufig ist ein Verfahrenswechsel in einer zweiten Sitzung notwendig.

Im Anschluss an die Skelettstabilisierung erfolgt die Versorgung der Sehnen. Sofern es möglich ist, werden sämtliche vollständig- oder teildurchtrennten Sehnen z.B. in der Nahttechnik nach KirchmayrKessler bzw. mittels U-Nähte versorgt. Ansatznahe Aus- oder Abrisse der tiefen Beugesehnen versorgen wir mittels einer Ausziehnaht. Im Bereich der dorsalen PIP-Gelenke ist zusätzlich auf den Verschluss der Strecksehnenaponeurose zu achten, um z.B. eine sekundäre Knopflochdeformität $\mathrm{zu}$ vermeiden. Bei der Versorgung der Beugesehnen ist der zeitliche Aspekt wesentlich. Ist es technisch möglich, sollten sowohl alle oberflächlichen als auch tiefen Beugesehnen versorgt werden. Wenn erforderlich, kann auf die Versorgung der oberflächlichen Beugesehnen verzichtet werden. Die nicht versorgten oberflächlichen Beugesehnen oder die Palmaris longus-Sehne können als Ersatzlager Verwendung finden. Bei nicht erhaltenem Gleitlager ist z.B. die Einlage eines Platzhalters in Form eines Silastikstabs (Spacer) eine Möglichkeit.

Wichtig ist bei der Beugesehnenversorgung im Fingerbereich die Schonung der vorhandenen Ringbänder.

Sind diese zerstört, so sind zumindest die Ringbänder A2 und A4 zu rekonstruieren, um einen Bogensehneneffekt zu vermeiden.
Erst jetzt erfolgt die mikrochirurgische Naht der Gefäße und Nerven. Ist genügend Zeit vorhanden, kann die Nervenversorgung vor der Gefäßnaht erfolgen, um den Vorteil der Blutleere auszunutzen. Im anderen Falle ist zuerst die Reperfusion in Form einer End-zu-End- oder End-zu-Seit-Anastomose wiederherzustellen. Bei nicht spannungsfrei zu nähenden Anastomosen und Defektverletzungen ist die Überbrückung mittels eines Veneninterponates Standard. Dieses kann z.B. aus nicht verwendbaren amputierten Körperteilen, dem Unterarm oder Fußrücken gewonnen werden. Eine weitere Möglichkeit ist auch die Anwendung eines Durchströmungslappens z. B. vom Fußrücken, bei gleichzeitig vorhandenen Weichteildefekten.

Als allgemeine Regel gilt, dass für jede Arterie zwei Venen versorgt werden sollten, um den venösen Abstrom sicher zu gewährleisten.

Die venöse Abflussbehinderung ist der häufigste Grund für sekundäre Verluste von replantierten Fingern. Intraoperativ beginnt die medikamentöse Unterstützung der Perfusion und Thromboseprophylaxe z.B. durch Gabe niedermolekularer Dextrane. Grundsätzlich sollten sämtliche Nervendurchtrennungen, sofern eine spannungsfreie epineurale Koaptation möglich ist, primär versorgt werden. Defektdurchtrennungen können primär durch Nerveninterponate rekonstruiert werden. Die Versorgung des Hautmantels ist bei komplexen Handverletzungen ein großes Problem. Zum einen müssen zum Überleben alle wichtigen funktionellen Strukturen von Weichteilen bedeckt sein, andererseits muss der Hautverschluss spannungsfrei erfolgen. Häufig verbleiben nach ausgiebigen Debridements Weichteildefekte zurück. Viele Möglichkeiten sind bisher zur Rekonstruktion des Weichteilmantels entwickelt worden. Zur Anwendung kommen Spalt- oder Vollhauttransplantate, lokale Lappenplastiken, gefäßgestielte Fernlappen bis hin zur freien Lappentransplantation. In der Literatur geht der Trend der Weichteildefektdeckung derzeit zum primären bzw. postprimären Verschluss durch Lappenplastiken innerhalb der ersten 72 Stunden $[8,11,3,9]$.

Voll- bzw. Spalthauttransplantate erfordern für das Anheilen ein gutes Wirtslager (z. B. gutes Granulationsgewebe). Die Deckung freiliegender Sehnen oder Knochen gelingt durch ein freies Hauttransplantat nicht. Die Vollhaut hat gegenüber 
der Spalthaut den Vorteil der besseren funktionellen und ästhetischen Ergebnisse. Die Spalthaut dagegen führt zu einem schnelleren und sicheren Einheilen. Im Falle von noch notwendigen sekundären Rekonstruktionseingriffen im Bereich eines großen Weichteildefektes entscheiden wir uns zugunsten einer vitalen Lappenplastik. In seltenen Fällen kann hier auch eine freie, vaskularisierte Lappenplastik infrage kommen (sog. „emergency flap“). Die Auswahl der Lappenplastiken richtet sich nach der Lokalisation, Beschaffenheit, Größe des Weichteildefektes und nach dem Patientenwunsch. Zur postoperativen Ruhigstellung der verletzten Hand wird eine gut gepolsterte Gipsschiene in IntrinsicPlus-Stellung angelegt.

\section{Die Nachbehandlung}

Unmittelbar postoperativ ist es wichtig, die verletzte Hand konsequent hochzulagern und engmaschig zu kontrollieren. Je nach Stabilität der Osteosynthese, der Art Sehnen- bzw. der Nervenversorgung erfolgt die Ruhigstellung der verletzten Hand in einer Gipsschiene.

Die Mobilisierung der betroffenen Strukturen ist so früh wie irgend möglich anzustreben. Dies kann unmittelbar postoperativ sein. Nach Replantationen und Transplantation von freien Lappenplastiken wird postoperativ ein wärmender und lockerer Verband angelegt, welcher z. B. eine Kompression im Bereich der Anastomosen verhindert. Die krankengymnastische Nachbehandlung richtet sich natürlich nach den Verletzungen. Dabei ist eine sofortige aktive und/oder passive Mobilisation sämtlicher nicht verletzter Gelenke, anzustreben, um Gelenkkontrakturen und Sehnenverklebungen zu vermeiden. Sehnenverletzungen werden, sofern keine Ruhigstellung aufgrund einer anderen betroffenen Struktur notwendig ist, dynamisch (z.B. nach Kleinert) nachbehandelt. Jede noch so kleine Funktionseinschränkung der Hand führt zu einer Schonung der gesamten Hand und des Armes. Der Patient muss deshalb dazu angehalten werden, von Anfang an alle nicht verletzten Gelenke zu bewegen. Nur so kann die Einsteifung auch dieser Gelenke vermieden werden und macht deutlich, dass sich die Nachbehandlung auf die gesamt Hand einschließlich des
Armes beziehen muss. Ist der Patient nicht in der Lage dies alleine durchzuführen, ist eine ergotherapeutische Behandlung auch der unverletzten Strukturen indiziert. Die Ergotherapie stellt eine wichtige Säule in der Behandlung der komplexen Handverletzung dar. Hier lernt der Patient Funktionseinschränkung seiner verletzten Hand im alltäglichen Leben umzugehen. Häufig sind die Patienten angenehm überrascht, was alles mit einer funktionsgestörten Hand noch möglich ist. Ein extrem positives Beispiel eines Gitarrenlehrers führt Neuber auf [7], welcher mit der linken Hand in eine Fräsmaschine geriet. Trotz einer erheblichen Funktionseinsschränkung dieser Hand arbeitete der Patient weiter als Gitarrenlehrer und war in der Lage weiterhin auf sehr hohem Niveau Gitarre zu spielen, wie gutachterlich festgestellt werden konnte. Dies zeigt, wie stark die soziale und berufliche Rehabilitation des Patienten von der Eigenmotivation abhängt. Dieses dem Patienten zu vermitteln, ist u. a. Aufgabe des behandelnden Arztes.

\section{Resüimee}

Aufgrund der meist schweren Weichteilverletzungen und der hohen Keimkontamination ist das initiale ausgiebige Debridement neben der qualitativ guten Versorgung der verletzten Strukturen entscheidend für das Out-come des Patienten. Nur so ist ein postoperativer Infekt zu vermeiden, welcher selbst die beste Versorgung zunichte machen würde. Wenn es möglich ist, sollte die komplexe Handverletzung primär definitiv versorgt werden. Allerdings ist nach unseren Erfahrungen die Vitalität der Weichteile nicht immer primär eindeutig zu erkennen, so dass wir primär nur solche verletzten Strukturen versorgen, welche eine sichere Heilungschance besitzen. Die Stabilisierung des Skeletts muss zur Vermeidung weiterer Weichteilschädigungen und aufgrund der meist zeitlichen Einschränkung minimalinvasiv, schnell durchführbar sein und muss eine frühfunktionelle Behandlung gewährleisten. Bei der Sehnenversorgung ist der zeitliche Aspekt mitentscheidend. Wenn es möglich ist, sollten primär sämtliche Sehnen versorgt werden. Eine Versorgung der tiefen Beugesehnen ist obligat.
Nervendurchtrennungen werden bei intakten Weichteilen primär koaptiert. Liegen zu versorgende Gefäßläsionen vor, so werden diese direkt oder bei langstreckigen Läsionen mittels Veneninterponat versorgt. Hinsichtlich der Versorgung von Weichteildefekten und fraglichen Gewebeuntergängen nehmen wir eher eine schnelle und aggressive Position und führen diese möglichst innerhalb von 72 Stunden durch. Die Versorgung einer komplexen Handverletzung stellt höchste Anforderungen an den behandelnden Hand- und Mikrochirurgen. Durch Erfahrung, Ideenreichtum und operatives Geschick kann aus einer primär hoffnungslos erscheinenden Handverletzung noch eine Grundlage geschaffen werden, auf die aufbauend eine ergound physiotherapeutische Behandlung und gegebenenfalls durch sekundäre Eingriffen ein gutes funktionelles und sensibles Ergebnis erzielt werden kann. Dies ermöglicht dem Patienten eine soziale und berufliche Reintegration. Nicht zu vernachlässigen ist auch die psychologische Führung des Patienten, ohne dessen engagierte Mithilfe die allerbeste Therapie nur einen Schatten ihrer selbst darstellt.

\section{Literatur}

${ }^{1}$ Berger A, Brenner P, Flory P, Schaller E, Schneider W. Progress in limb and digital replantation. Part. B. World J Surg 1990; 14: 807-818

${ }^{2}$ Biemer E. Indikation und Grenzen der Replantation. Chirurg 1990; 61: $103-108$

${ }^{3}$ Brenner P, Becker M, Lassner F, Berger A. Zeitpunkt der freien mikrochirurgischen Gewebetransplantation bei Handverletzungen in der Akutphase. Handchir Mikrochir Plast Chir 1995; 27: 286-291

${ }^{4}$ Chen Z-W, Meyer VE, Kleinert HE, Beasley RW. Present indications and contraindications for replantation as reflected by longterm funktional results. Orthop Clin North Am 1981; 12: 849-870

${ }^{5}$ Germann G, Karle B, Brüner S, Menke H. Behandlungsstrategie bei komplexen Handverletzungen. Unfallchirurg 2001; 103 : $342-347$

${ }^{6}$ Maschwitz B. Anforderungen an die Begleitund Nachbehandlung in der Physiotherapie bei komplexen Handverletzungen. Trauma Berufskrankh 1999; 1 (Suppl 1): 67-72

${ }^{7}$ Neuber M, Joist A, Probst A. Patientenmotivation - unverzichtbare therapeutische Hilfe. Chirurg 1998; 69: $581-584$

${ }^{8}$ Ninkovic M, Hussl H, Hefel L, Anderl H. Zeitpunkt der Versorgung schwerer Verletzungen der oberen Extremität durch freie Lappen. Handchir Mikrochir Plast Chir 1995; 27: $297-306$ 
${ }^{9}$ Pechlaner S, Hussl H. Das Komplextrauma der Hand. Orthopäde 1998; 27: $11-16$

${ }^{10}$ Schaller HE. Operatives Vorgehen bei der Akutversorgung von schweren Handverletzungen und der plastischen Deckung bei großen Defekten. Trauma Berufskrankh 1999; 5: 59-66
${ }^{11}$ Schwabegger AH, Anderl H, Hussl H, Ninkovic M. Komplexe Handverletzungen - Stellenwert der Primärversorgung mit freien Lappen. Unfallchirurg 1999; 102: 292-297 ${ }^{12}$ Wilgis E, Shaw F. Replantation. IN: Neviaser R.J. (Ed.) Controversies in Hand Surgery. Churchill Livingstone, New York 1990

\section{Dr. med. Alexander Zach}

Assistenzarzt in der Abteilung für Hand-, Replantations- und Mikrochirurgie Prof. Dr. med. Axel Ekkernkamp Ärztlicher Direktor und Geschäftsführer Priv.-Doz. Dr. med. Andreas Eisenschenk Chefarzt der Abt. für Hand-, Replantations- und Mikrochirurgie

Unfallkrankenhaus Berlin-Marzahn mit Berufsgenossenschaftlicher Unfallklinik e.V.

Warener Str. 7

12683 Berlin 